\title{
Comparación de uniformidades de distribución en riegos con incremento de gasto y continuo en surcos cerrados
}

\author{
E. Vázquez-Fernández, P. López-Tellez y B. Chagoya-Amador \\ Instituto de Ingeniería, UNAM \\ E-mail: ernesvf@ servidor.unam.mx
}

(recibido: junio de 2003; aceptado: octubre de 2003)

\begin{abstract}
Resumen
Se presenta un análisis comparativo entre las uniformidades de distribución de las láminas de agua infiltradas en surcos cerrados, después de la aplicación de tres riegos con incremento de gasto y tres con gasto constante 0 riego continuo, con el objetivo de evaluar al primero. La comparación con este último es porque aún es el más empleado en los países en desarrollo. El promedio de las uniformidades de distribución del primer método es supe rior en $9 \%$ al del segundo; por tanto, se concluye que el riego con incremento de gasto es una opción viable de riego eficiente en surcos cerrados que, en comparación con el riego continuo, es alternativo a éste para producir ahorros de agua del orden de $8 \%$. Las pruebas de campo se realizaron en surcos de $290 \mathrm{~m}$ de longitud, $75 \mathrm{~cm}$ de ancho, $0.6 \%$ de pendiente y textura franco-arcillosa.
\end{abstract}

Descriptores: riego continuo, etapa de avance del agua, riego con incremento de gasto, uniformidad de distribución.

\begin{abstract}
In this pa per wepresent a com par a tiveanal y sisbetween uni for mi ties of distri bu tion of wa ter depths in fil trated in blocked-end fur rows, af ter thetrial of threeir ri ga tion with thein crease dis chargeand threewith con stant dis chargeor con tin u ous ir ri ga tion with theob jectiveof eval u at ing thefor mer. Thecom par i son with thelast oneis becauseit is themost used onein coun tries in devel op ment. Theaver ageof theuni for mi ties of thedis tri bu tion of thefirst method ishigher in $9 \%$ regard ing thesec ond one, thus, we con cludethat their ri ga tion with in creasedis charge is a vi a bleop tion of ef fi cient ir ri ga tion in blocked-end furrows that, in com par i son with continuous ir ri ga tion, is an al ter na tivein or der to get saving of wa ter of about $8 \%$. Fields tri als werereal ized in blocked-end fur rows of 290 meters $10 n g, 75 \mathrm{~cm}$ wide, $0.6 \%$ de clineand clay-loam tex ture.
\end{abstract}

Keywords: con tin u ousir ri ga tion, ad vancephase, clay-loam, ir ri ga tion with in crease discharge, distribution uni formity. 
Comparación de uniformidades de distribución en riegos con incremento de gasto ...

\section{Introducción}

La escasez de agua en los países en desarrollo representa actualmente una gran preocupación de los gobiernos, por lo que el uso eficiente del agua de riego es, en algunos de ellos, la prioridad número uno para la conservación del agua. En México, por ejemplo, en más del $80 \%$ de la superficie regada los métodos son tradicionales, y como el porcentaje dedicado a la agricultura es del $78 \%$ del total de agua aprovechado, resulta que el $62.4 \%(0.80 * 78 \%)$ de este total es empleado en el riego tradicional, con una eficiencia de aplicación estimada en las parcelas del 59\% (Alexander-Frezieres, 2001). Como la gran la mayoría de las parcelas que se riegan por gravedad tienen los surcos cerrados en su parte más baja, resulta que se trata de una eficiencia muy pequeña, pues al evitarse el es cape del agua se podrían alcanzar eficiencias mayores, como se comprueba de manera colateral en este trabajo, aún con el riego continuo que es el menos eficiente de los métodos de riego por surcos.

La búsqueda de métodos eficientes de riego por surcos que sustituyan al método tradicional de riego con tinuo ha dado lugar a la creación de los métodos cutback e intermitente; éste último es el más eficiente, pero requiere del empleo de válvulas y controladores electrónicos costosos que muchos agricultores de los países en desarrollo no pueden adquirir.

El procedimiento del riego con incremento de gasto en surcos cerrados se describe en Vázquez y Gracia (2002) y es el inverso del riego con recorte 0 cutback propuesto por Humpherys en Jensen (1980). El primero se aplica, inicialmente, como en el riego continuo, con un gasto constante en toda la batería de surcos, la cual conviene que tenga un número par de ellos; antes de que se termine la fase de avance del agua, que es el tiempo que tarda en llegar ésta a la parte final de los surcos, el gasto total de alimentación se dirige a una mitad de la batería de surcos, de tal forma que se duplica y se corta al infiltrar la lámina de agua que se desea; enseguida, todo el cau dal se dirige a la otra mitad de la batería de surcos, donde también se duplica el gasto; y para concluir se iguala el tiempo de riego otorgado a la primera mitad.

El método ha mostrado ser alternativo a los riegos con tinuo e intermitente en la teoría, con la ventaja de que no utiliza válvulas electrónicas. En trabajos previos, las eficiencias de aplicación teóricas del riego con incremento de gasto fueron comparadas con el cutback (Vázquez y Gracia, 2002), donde las eficiencias son aproximadamente iguales, siendo que éste ha sido probado en campo desde hace muchos años como uno de los más eficientes del riego por surcos (Martínez-Elizondo, 1980); (Walker y Skogerboe, 1987).

En una primera evaluación en el campo (Vázquez et al., 2003) se compararon las uniformidades de distribución de dos riegos con incremento de gasto y dos con intermitente, siendo los resultados promedio muy aproximados. La Uniformidad de Distribución (UD) se de fine como la relación de la lámina mínima infiltrada en algún lugar del surco entre la lámina media infiltrada a lo largo del mismo (Burt et al., 1997). Ahora se presenta una segunda evaluación, también con mediciones de campo de las UD, entre el riego con incremento de gasto y el riego continuo, con el objetivo de verificar que sea alternativo a este último. 


\section{Materiales y métodos}

Los surcos de prueba tienen las siguientes características: longitud de $290 \mathrm{~m}$, pendiente de $0.6 \%$, suelo franco-arcilloso (conductividad hidráulica de $0.018 \mathrm{~cm} / \mathrm{min}$ ) y una separación entre surcos igual a $75 \mathrm{~cm}$. En la aplicación de los riegos se utilizó una tubería de PVC con compuertas, esto permitió que en el riego con incremento de gasto la operación de ellas fuera manual, sin necesidad de emplear una válvula electrónica.

Las mediciones de la humedad del suelo se realizaron con el método gravimétrico para profundidades de 0 a $30 \mathrm{~cm}$ y 30 a $60 \mathrm{~cm}$ durante el ciclo de cultivo de la zanahoria. En los riegos aquí notificados, en seis de un total de nueve, se midieron las humedades del suelo antes de iniciarlos 48 ó 72 horas después de la aplicación. En los primeros tres riegos con incremento de gasto no se realizaron mediciones de las humedades. La toma de muestras tuvo lugar en al menos tres de los siguientes sitios medidos a partir de la cabecera de los surcos 50, 100, 190 y $240 \mathrm{~m}$.

Las láminas de agua infiltradas se calculan con la ecuación

$$
Z=h \rho_{r}\left(\frac{W_{f}-W_{i}}{100}\right)
$$

donde $Z$ es la lámina de agua infiltrada en $\mathrm{cm}$; $\mathrm{h}=60 \mathrm{~cm}$, profundidad máxima de medición de la humedad; $W_{f}$ la humedad del suelo medida 24 ó 72 horas después del riego en \% de peso seco; $W$ humedad inicial del suelo antes del riego en $\%$ de peso seco; $?_{r}=1.35$, densidad relativa aparente del suelo, adimensional.

Las muestras de humedad del suelo en los riegos con incremento de gasto se tomaron en surcos representativos de la primera mitad de la batería de surcos, la cual se considera más desfavorable para la medición de la eficiencia por no presentar discontinuidad en la aplicación del riego, como es el caso de la segunda mitad de la batería de surcos (Figura 1) que tiene semejanza con el riego intermitente en el primer ciclo. En la figura 1, $Q_{0}$ es el gasto inicial, $T_{i}$ el tiempo de incremento de este gasto en la primera batería de surcos; $T_{m}$ inicio del riego complementario en la segunda mitad de la batería de surcos y

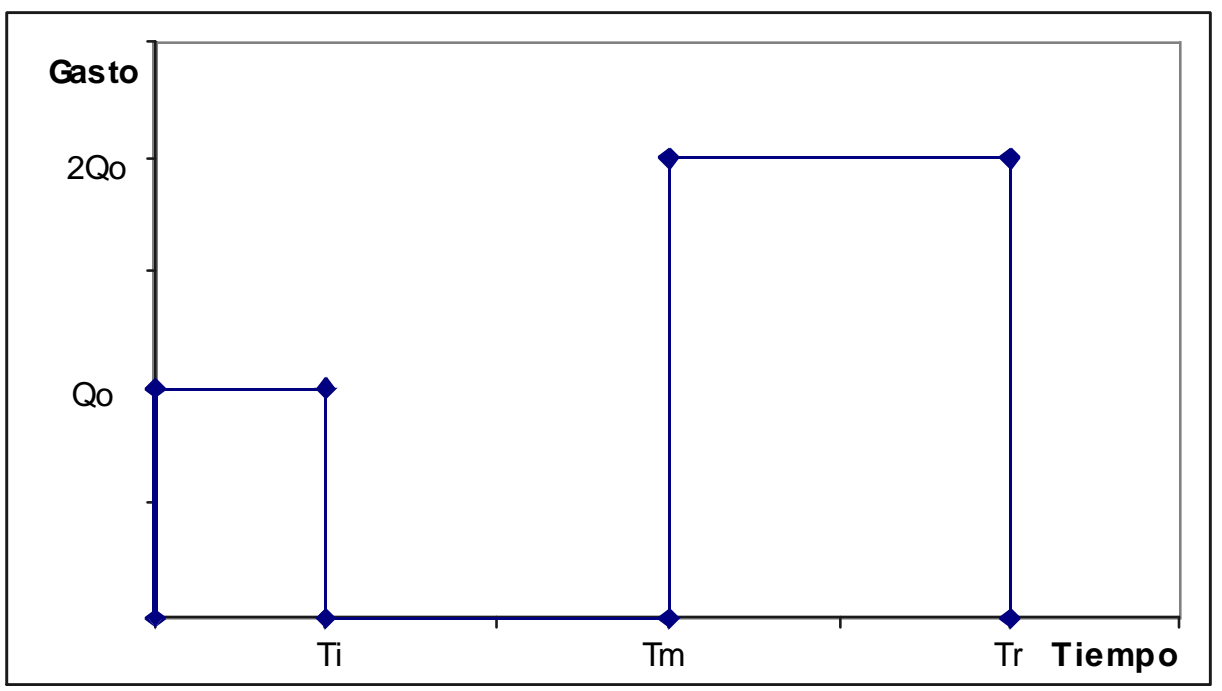

Figura 1. Hidrograma de ingreso a la segunda mitad de la batería de surcos 
tiempo de riego de la primera, $T_{r}$ el tiempo de riego en toda la batería de surcos.

\section{Resultados}

En la figura 2 se muestra el hidrograma tipo ingreso correspondiente a la primera mitad de la batería de surcos, mediante el método de riego con incremento de gasto. La notación, excepto la duración de la etapa de avance del agua, $T_{a}$, que se encuentra entre $T_{i}$ y $T_{m}$, coincide con la ecuación empleada para calcular el volumen

$$
V_{e}=0.060\left[Q_{0} T_{i}+2 Q_{0}\left(T_{m}-T_{i}\right)\right]
$$

donde $V_{e}$ es el volumen de ingreso al surco en $\mathrm{m}^{3} ; \mathrm{Q}_{0}$ el gasto inicial en $\mathrm{L} / \mathrm{s} ; \mathrm{T}_{i}$ el tiempo de incremento del gasto inicial en $\mathrm{min} ; \mathrm{T}_{\mathrm{m}}$ el tiempo de riego en la primera mitad de la batería de surcos en min.

En la tabla 1 se anotan los gastos, tiempos, la fracción de longitud del surco para la cual se realizó el incremento de gasto, volúmenes de ingreso a los surcos y láminas de aplicación o lámina bruta para los diferentes riegos. Las siglas RIG y RC corresponden a los riegos con

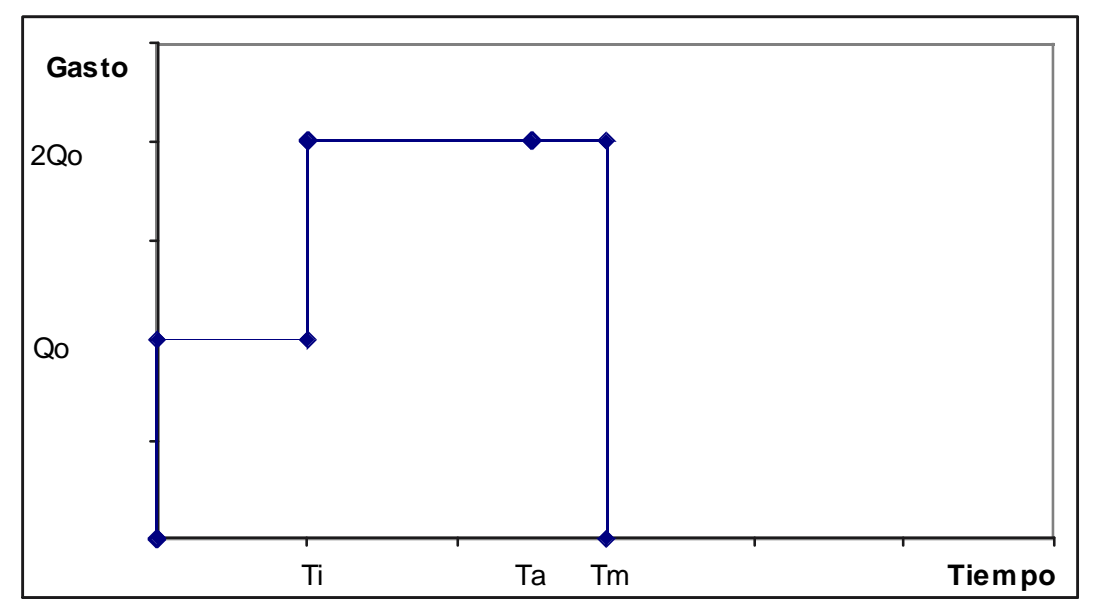

Figura 2. Hidrograma de ingreso para la primera mitad de la batería de surcos

Tabla 1. Gasto, tiempo y volúmenes de ingreso de los riegos

\begin{tabular}{|c|c|c|c|c|c|c|c|}
\hline $\begin{array}{l}\text { Tipo y } \\
\text { numero } \\
\text { de riego }\end{array}$ & $\begin{array}{l}\text { Gasto Inicial } \\
Q_{0}[\mathrm{~L} / \mathrm{s}]\end{array}$ & $\begin{array}{l}\text { Tiempo de } \\
\text { incremento de } \\
\text { gasto } \mathrm{T}_{\mathrm{i}}[\mathrm{min}]\end{array}$ & $\begin{array}{c}\text { Duración de la } \\
\text { etapa de } \\
\text { avance } \\
\text { [min] }\end{array}$ & $\begin{array}{l}\text { Tiempo de } \\
\text { riego } \text { Tr }_{\mathrm{r}}[\mathrm{min}]\end{array}$ & $\begin{array}{l}\text { Fracción de } \\
\text { longitud del } \\
\text { surco en el } \\
\text { incremento }\end{array}$ & $\begin{array}{l}\text { Volumen de } \\
\text { ingreso } \mathrm{V}_{e}\left[\mathrm{~m}^{3}\right]\end{array}$ & $\begin{array}{c}\text { Lámina de } \\
\text { riego bruta } \\
\text { [cm] }\end{array}$ \\
\hline RIG-1 & 2.375 & 78 & 78 & 94 & 1.00 & 15.675 & 7.21 \\
\hline RIG-2 & 0.66 & 23 & 47 & 120 & 0.57 & 8.593 & 3.95 \\
\hline RIG-3 & 0.95 & 26 & 46 & 74 & 0.52 & 6.498 & 2.99 \\
\hline $\mathrm{RC}-4$ & 1.54 & - & 50 & 90 & - & 8.316 & 3.82 \\
\hline $\mathrm{RC}-5$ & 1.03 & - & 240 & 240 & - & 14.832 & 6.82 \\
\hline RIG- 6 & 1.336 & 30 & 130 & 150 & 0.25 & 21.643 & 9.95 \\
\hline RIG-7 & 0.584 & 56 & 244 & 244 & 0.25 & 15.137 & 6.96 \\
\hline $\mathrm{RC}-8$ & 1.12 & - & 216 & 216 & - & 14.515 & 6.67 \\
\hline RIG-9 & 0.895 & 26 & 126 & 140 & 0.25 & 13.151 & 6.05 \\
\hline
\end{tabular}


incremento de gasto y con tinuo, respectivamente.

En la tabla 2 se presentan las fechas de riego y las temperaturas medias mensuales.

En las figuras 3 a 4 se muestran las curvas de avance del agua para los riegos. La figura 3 corresponde al RC y la figura 4 al RIG. Las mediciones se tomaron cada $20 \mathrm{~m}$.
Las tab las 3, 4 y 5 muestran las humedades antes y después de los riegos, así como las láminas de agua calculadas con la ecuación 1 y los datos de las tablas 3 y 4 para profundidades de 0 a $60 \mathrm{~cm}$, respectivamente. Se agregan en la tabla 5 los volúmenes de agua infiltrados; nótese que los valores son menores a los de ingreso en la tabla 1.

Tabla 2. Fechas de riego y temperaturas medias mensuales

\begin{tabular}{|c|c|c|c|c|c|}
\hline Riego número & Fecha & Diferencia en días & Días acumulados & $\begin{array}{c}\text { Temperatura media } \\
\text { mensual }\left[{ }^{\circ} \mathrm{C}\right]\end{array}$ & Método de riego \\
\hline & Nov-16-2002 & 0 & & 12.8 & (Siembra) \\
\hline 1 & Nov-19-2002 & 3 & 3 & $“$ & RIG (sin muestreo) \\
\hline 2 & Nov-30-2002 & 11 & 14 & $“$ & RIG (sin muestreo) \\
\hline 3 & Dic-10-2002 & 10 & 24 & 11.5 & RIG (sin muestreo) \\
\hline 4 & Dic-24-2002 & 14 & 38 & $“$ & $\mathrm{RC}$ \\
\hline 5 & Ene-16-2003 & 23 & 61 & 11.4 & $\mathrm{RC}$ \\
\hline 6 & Feb-13-2003 & 28 & 89 & 13.0 & RIG \\
\hline 7 & Mar-06-2003 & 21 & 110 & 15.5 & RIG \\
\hline 8 & Mar-21-2003 & 15 & 125 & $“$ & $\mathrm{RC}$ \\
\hline 9 & Mar-27-2003 & 6 & 131 & “ & RIG \\
\hline
\end{tabular}

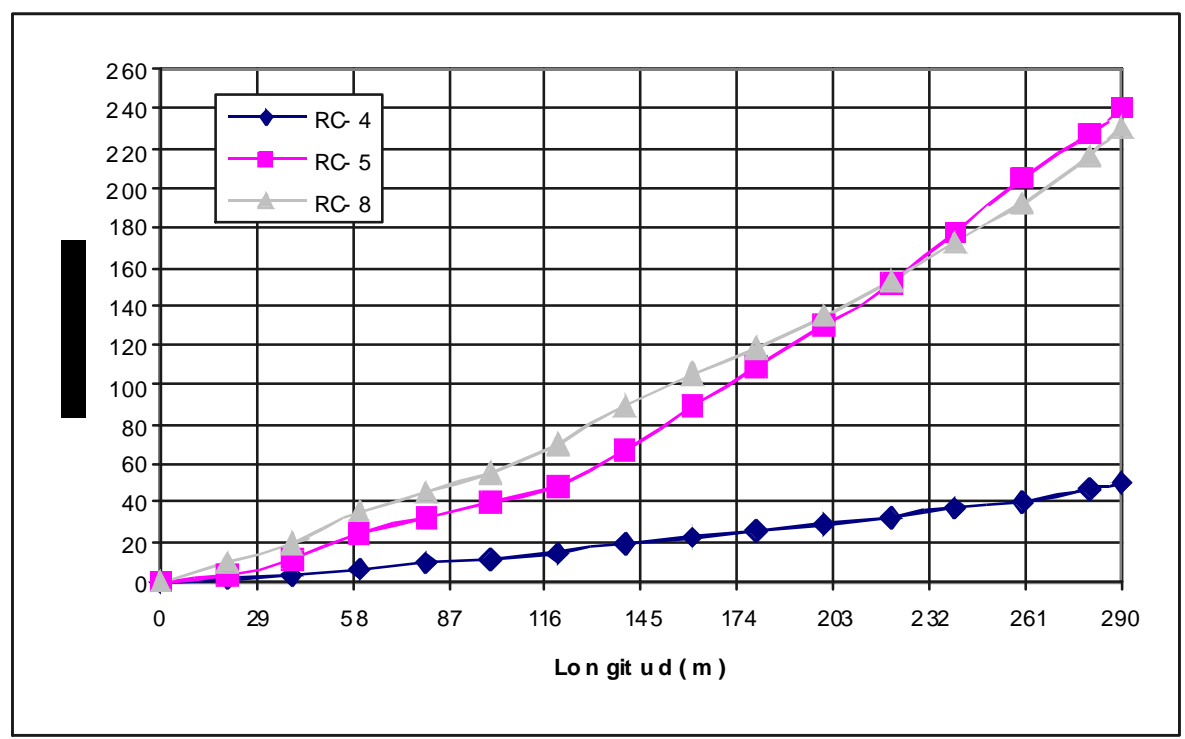

Figura 3. A vance del agua, RC-4, RC-5, RC-8 
Comparación de uniformidades de distribución en riegos con incremento de gasto ...

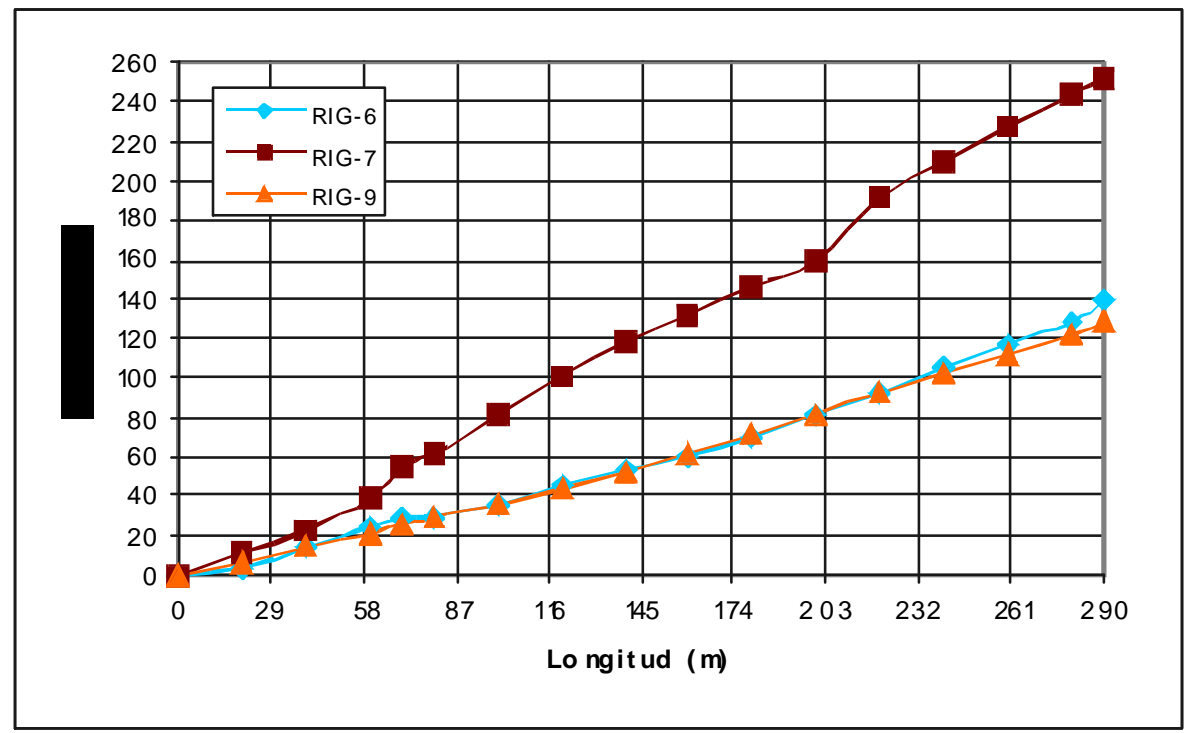

Figura 4. A vance del agua, RIG-6, RIG-7, RIG-9

La figura 5 muestra la distribución de las láminas de agua infiltradas a lo largo del surco para los riegos con incremento de gasto y la figura 6 para los riegos con gasto constante.

Tabla 3. Humedades del suelo antes de los riegos [\% en peso seco]

\begin{tabular}{ccccccc}
\hline \multirow{2}{*}{$\begin{array}{c}\text { Riego } \\
\text { número }\end{array}$} & \multicolumn{5}{c}{ Distancia en el surco [m] } & $\begin{array}{c}\text { Promedio } \\
\text { en el surco }\end{array}$ \\
\cline { 2 - 6 } & 50 & 100 & 145 & 190 & 240 & 22.658 \\
RC-4 & 22.62 & 21.02 & & 22.03 & 24.96 & 20.534 \\
RC-5 & 19.53 & & 19.62 & & 22.46 & 16.200 \\
RIG-6 & 15.04 & & 16.44 & & 17.12 & 20.043 \\
RIG-7 & 18.93 & & 19.26 & & 21.94 & 18.745 \\
RC-8 & 17.89 & 18.57 & & 19.04 & 19.48 & 22.155 \\
RIG-9 & 21.63 & 20.95 & & 22.80 & 23.24 & \\
\hline
\end{tabular}

Tabla 4. Humedades del suelo 48 y $72 *$ horas después de los riegos [\% en peso seco]

\begin{tabular}{ccccccc}
\hline \multirow{2}{*}{$\begin{array}{c}\text { Riego } \\
\text { número }\end{array}$} & \multicolumn{5}{c}{ Distancia en el surco [m] } & $\begin{array}{c}\text { Promedio } \\
\text { en el surco }\end{array}$ \\
\cline { 2 - 6 } & 50 & 100 & 145 & 190 & 240 & 26.748 \\
RC-4 & 26.58 & 24.96 & & 27.03 & 28.42 & 28.243 \\
RC-5* & 26.22 & & 28.66 & & 29.85 & 27.390 \\
RIG-6 & 27.22 & & 27.07 & & 27.88 & 29.053 \\
RIG-7 & 27.46 & & 29.80 & & 29.90 & 26.240 \\
RC-8* & 25.62 & 24.81 & & 27.00 & 27.53 & 28.915 \\
RIG-9 & 27.69 & 28.65 & & 29.12 & 30.20 & \\
\hline
\end{tabular}


E. Vázquez-Fernández, P. López-Tellez y B. Chagoya-Amador

Tabla 5. Láminas de agua infiltradas [cm]

\begin{tabular}{|c|c|c|c|c|c|c|c|c|}
\hline \multirow{2}{*}{$\begin{array}{l}\text { Riego } \\
\text { número }\end{array}$} & \multicolumn{5}{|c|}{ Distancia en el surco [m] } & \multirow{2}{*}{$\begin{array}{l}\text { - Promedio en } \\
\text { el surco }\end{array}$} & \multirow{2}{*}{ UD } & \multirow{2}{*}{$\begin{array}{l}\text { Volumen } \\
\text { infiltrado }\left[\mathrm{m}^{3}\right]\end{array}$} \\
\hline & 50 & 100 & 145 & 190 & 240 & & & \\
\hline RC-4 & 3.21 & 4.00 & & 4.05 & 2.80 & 3.515 & 0.796 & 7.644 \\
\hline $\mathrm{RC}-5^{*}$ & 5.42 & & 7.32 & & 5.98 & 6.239 & 0.868 & 13.570 \\
\hline RIG-6 & 9.87 & & 8.61 & & 8.71 & 9.063 & 0.950 & 19.712 \\
\hline RIG-7 & 6.91 & 7.49 & & 5.90 & 5.64 & 6.486 & 0.870 & 14.106 \\
\hline $\mathrm{RC}-8^{*}$ & 6.26 & 5.06 & & 6.45 & 6.52 & 6.073 & 0.833 & 13.208 \\
\hline RIG-9 & 4.91 & 6.23 & & 5.12 & 5.64 & 5.472 & 0.896 & 11.902 \\
\hline
\end{tabular}

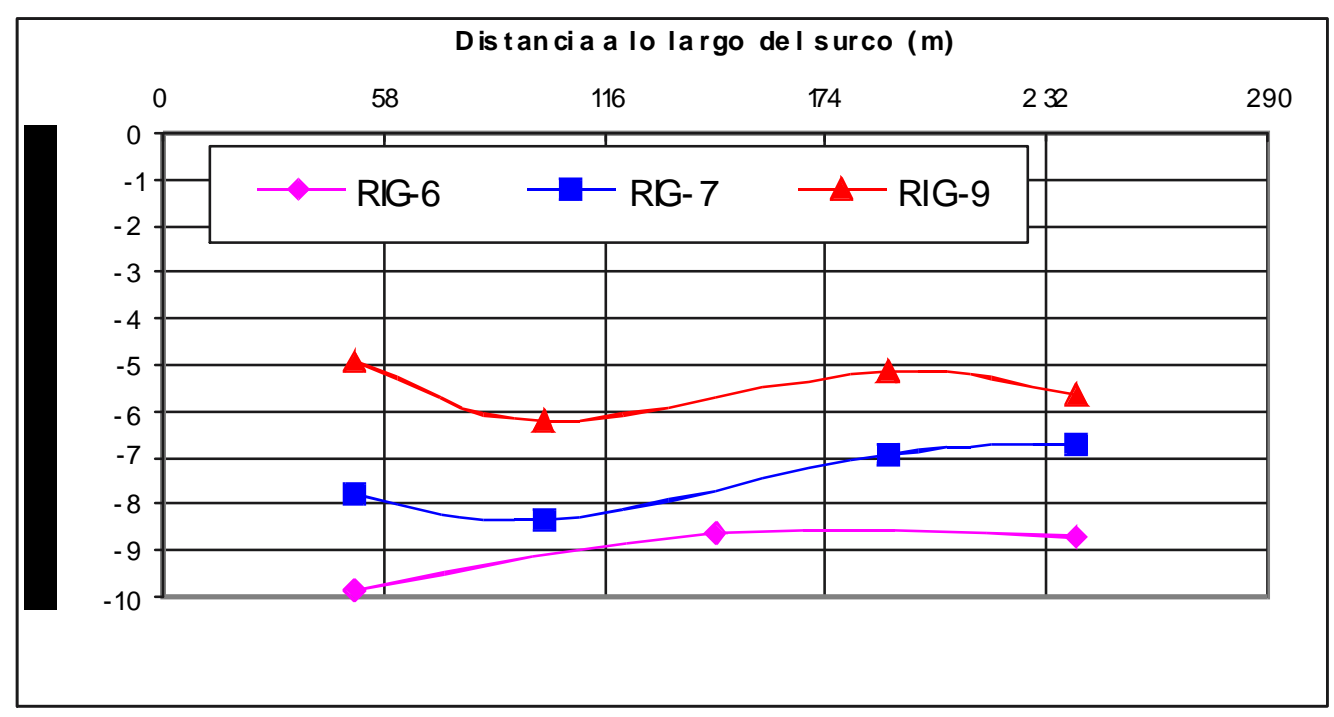

Figura 5. Distribución de las láminas de agua infiltradas en los RIG

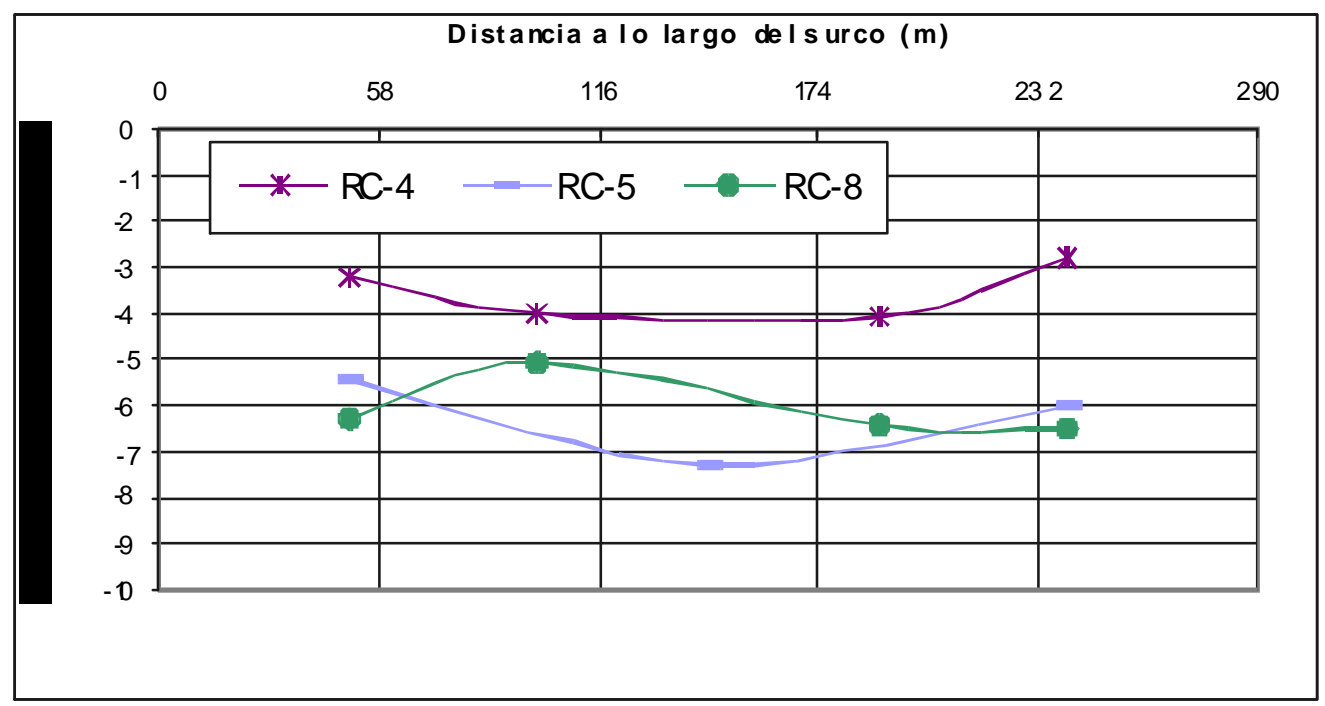

Figura 6. Distribución de las láminas de agua en los RC 
Comparación de uniformidades de distribución en riegos con incremento de gasto ...

\section{Discusión}

No obstante que los riegos con los dos métodos aquí contrastados no se hayan aplicado en forma simultánea, se considera válido comparar las uniformidades de distribución de ambos para evaluar el riego con incremento de gasto y decidir si es alternativo al riego con tinuo.

En la tabla 1 se suma una lámina bruta de riego igual a $54.42 \mathrm{~cm}$. En otro cultivo de zanahoria en la misma parcela y ciclo otoño-invierno se aplicó una lámina de $89 \mathrm{~cm}$ con riego con tinuo (López-López, 1991), por lo que el ahorro de agua fue de $39 \%$. Sin embargo, no se comparó la producción de zanahoria en tamaño y peso porque el cultivo desarrollado du rante los nueve riegos con RIG y RC de esta investigación se dejó en el campo como abono verde.

Nótese que en las curvas de avance de la figura 3 se presentan las curvas exponenciales ascendentes tradicionales del RC; en cambio, en las de la figura 4 correspondientes al RIG los puntos se ajustan aproximadamente a una recta desde el origen, lo que reduce la duración de la etapa de avance del agua, en comparación con un gasto continuo igual al valor inicial. Esta reducción en tiempo es posible porque el incremento de gasto se realizó cuando el agua llegó a un cuarto de la longitud del surco en los tres riegos con incremento de gasto, provocándose un avance más rápido del agua con mayor cau dal sobre el suelo mojado y el frente de onda. En la primera evaluación del RIG (Vázquez et al., 2003) el incremento de gasto se realizó cuando el agua alcanzó la mitad de un surco con una longitud de $160 \mathrm{~m}$ y misma pendiente, obteniéndose una UD $=0.79$. Al ser mayor ahora las UD (Tabla 5) en un surco más grande, se deduce que es preferible el incremento a un cuarto de la longitud del surco, resultado que puede adoptarse como receta para cualquier longitud. Obsérvese en la tabla 1 que en los tres primeros riegos, los cuales sirvieron de preparación para la aplicación de los siguientes con el mismo método, la fracción de longitud del incremento es mayor que 0.25 .

Cabe destacar que esta recomendación de incrementar el gasto cuando el agua alcance un cuarto de la longitud del surco es similar a la empleada en el riego intermitente, donde la batería de surcos también se divide en mitades, para determinar el ciclo de activación de la válvula electrónica para el mojado de las mitades, es decir, es igual al tiempo de avance del agua hasta una cuarta parte de la longitud del surco (www.pyrargentina.com.ar).

En las figuras 5 y 6 se observa que en dos de los tres riegos la diferencia en las láminas infiltradas se encuentra al inicio de los surcos, con láminas infiltradas mayores en el RIG que en el RC, excepto en el RIG-9. Este último resultado se explica porque el riego se aplicó a sólo seis días del RC-8, ya que fue con fines demostrativos para ilustrar el procedimiento del método.

Debe destacarse en la tabla 5 que las UD son mayores para los RIG que para los RC; las UD promedio de los RIG es 0.905 y de los RC es 0.832 .

Es probable que la distribución de las humedades en la segunda mitad de la batería de surcos no sea igual a las aquí presentadas para la primera. En un trabajo previo (Vázquez et al ., 2003) se tomaron muestras en la segunda 
mitad de la batería de surcos con resultados de las UD muy próximos a las del riego intermitente, por lo que en esta ocasión, al hacer la comparación con el RC se eligió la primera mitad.

El manejo del agua para aplicar el método es muy sencillo mediante el empleo de las tuberías con compuertas múltiples; sin embargo, también sería posible aplicarlo cuando se hace la alimentación directa a los surcos con boquillas (riego no tecnificado), tal como se practica en muchos países no desarrollados.

De acuerdo con las UD obtenidas en este trabajo, el ahorro de agua (Ag) se calcula como

$$
A g=(10.832 / 0.905) 100=8 \%
$$

Este ahorro, que no es muy significativo porque el RC fue bien aplicado, es suficiente para recomendar el empleo del RIG en vez del RC. Ahora bien, si la comparación se hiciera con las eficiencias de los riegos tradicionales aplicados en países en desarrollo (0.59 en México), el valor de $\mathrm{Ag}$ calculado con la ecuación 3 para México sería del 35\%, por lo que el impacto en el ahorro de agua sería no ta ble y con costos menores a los del riego intermitente.

\section{Conclusiones}

De acuerdo con la comparación de las uniformidades de distribución del riego con incremento de gasto (0.905) y el promedio de las mismas para los riegos con gasto constante (0.832), se concluye que el riego con incremento de gasto es factible de ser empleado en vez del riego continuo, con un ahorro de agua aproximado del $8 \%$.
El incremento 0 duplicación del gasto realizado cuando el frente de onda del agua alcanza un cuarto de la longitud del surco, permite que la duración de la etapa de avance se reduzca, porque el agua con el gasto incrementado viaja más rápido sobre suelo mojado, lográndose una mejor uniformidad de distribución.

El procedimiento para aplicar el riego con incremento de gasto en surcos cerrados no ofrece mayores dificultades si se emplea una tubería con compuertas en la cabecera de los surcos.

Es recomendable llevar al cabo otras evaluaciones en surcos con pendientes menores a $0.6 \%$ y aplicar otros métodos en forma simultánea al riego con incremento de gasto, tales como el de recorte o cutback e intermitente, con el fin de explorar la posibilidad de que también sea alternativo a éstos. En estos ensayos conviene realizar el incremento a un cuarto, 0 una relación menor de la longitud del surco con el fin de incrementar la uniformidad de distribución.

\section{Agradecimientos}

AI MC René Martínez Elizondo por facilitar los surcos de prueba en los campos experimentales de la Universidad Autónoma Chapingo. Al Dr. Jesús Gracia Sánchez por la revisión crítica del escrito.

\section{Referencias}

Alexander-Frezieres J. (2001). Conservación de la infraestructura hidroagrícola en las unidades de riego en México. Memorias del XI Congreso Nacional de Irrigación. Asociación Nacional de Especialistas en Irrigación. 
Burt C.M., Clemens A.J., Strelkoff T.S. Solomon K.H., Bliesner R.D., Hardy L.A., Howell T.A. y Eisenhauer D.E. (1997). Irrigation Performance Measures: Efficiency and Uni -formity. Journal of Irrigation and Drainage Engineering, Vol. 123, No. 6, p. 431.

Jensen M.E. ed. (1980). Design and 0 peration of Farm Irrigation Systems. American So ciety of Agri cul tural Engi neers, p. 569. López-López C. (1991). Evaluación para estimar el consumo de agua por la zanahoria (Daucus Carota L.) con la metodología de la FAO. Tesis de licenciatura, Universidad Autónoma Chapingo.

Martínez-Elizondo R. (1980). Metodologías para diseño de riego en surcos con reducción de gasto. Colegio de Postgraduados, Tesis de maestría.

Vázquez E. y Gracia J. (2002). Eficiencias de aplicación teórica en los métodos de riego con incremento de gasto y recorte en surcos cerrados. XX Congreso Latinoamericano de Hidráulica, La Habana, Cuba.

Vázquez E., López P. y Morales E. (2003). Primera evaluación en campo del riego con incremento de gasto en surcos cerrados. Memorias del XI Congreso Nacional de Irrigación, Zacatecas, Zac. Asociación Nacional de Especialistas en Irrigación.

Walker W.R., Skogerboe G.V. (1987). Surfacelrrigation. Prentice-Hall, p. 144. www.pyrargentina.com.ar. Hoja técnica No. 1. Riego por caudal discontinuo.

\section{Semblanza de los autores}

Ernesto Vázquez-Fernández. Egresado de la UNAM, ha sido profesor de la Universidad Juárez Autónoma de Tabasco y de la División de Estudios de Posgrado de la Facultad de Ingeniería, UNAM. Asimismo, se ha desempeñado como especialista en ingeniería hidráulica en los sectores público y privado. Actualmente labora en el Instituto de Ingeniería de la UNAM.

Pedro López Tellez. Egresado de la Universidad Autónoma de Chapingo, ha laborado como especialista en irrigación y asesor técnico agrícola en los sectores público y privado. Actualmente es pasante de maestría en ingeniería hidráulica en la División de Estudios de Posgrado de la Facultad de Ingeniería, UNAM.

Bernardino Chagoya-Amador. Es pasante de ingeniería civil por la Facultad de Ingeniería, UNAM. Actualmente es becario del Instituto de Ingeniería. Desarrolló su tesis de licenciatura sobre el mismo tema del artículo presentado. 